\title{
Relationship between the genetic expression of WTAP and bladder cancer and patient prognosis
}

\author{
LEZHONG CHEN $^{1,2}$ and XINGHUAN WANG ${ }^{1}$ \\ ${ }^{1}$ Department of Urology, Zhongnan Hospital of Wuhan University, Wuhan, Hubei 430071; \\ ${ }^{2}$ Department of Urology, Baoan Central Hospital of Shenzhen, Shenzhen, Guangdong 518102, P.R. China
}

Received December 28, 2017; Accepted July 9, 2018

DOI: $10.3892 / \mathrm{ol} .2018 .9554$

\begin{abstract}
The relationship between the gene expression of Wilms tumor 1-associated protein (WTAP) and bladder cancer was investigated to study the effect of its expression on patient prognosis. Sixty-two fresh specimens of bladder transitional cell cancer tissues were collected as the bladder cancer group, while 20 normal bladder mucosa specimens comprised the control group. Hematoxylin and eosin staining was conducted to detect the pathological differences between the groups and the immunohistochemistry was used to test the expression levels of WTAP in the tissues. Reverse transcription-polymerase chain reaction (RT-PCR) was used to detect the mRNA expression levels of WTAP. Moreover, western blot analysis was used to examine the WTAP expression levels. At the same time, Cox regression multi-factor survival analysis was conducted for the related factors to the prognoses of bladder cancer patients. The structures of cells in the bladder cancer group were destroyed as was evident by the shrunken cell nuclei, while the tissues in the control group were intact. WTAP expression in the bladder cancer group was significantly increased compared with that in the control group. A small number of mRNAs and proteins were higher in the bladder cancer group than that in the control group. The differences in WTAP expression between the bladder cancer and control groups were statistically significant $(\mathrm{P}<0.05)$. There were obvious differences in the postoperative recurrence risk between the patients with a negative WTAP protein expression and those with a positive one $(\mathrm{P}<0.05)$. In conclusion, WTAP may play an important role in the occurrence and development of bladder cancer and can be considered as a potential target for bladder cancer treatment, providing a new basis for clinical diagnoses.
\end{abstract}

Correspondence to: Dr Xinghuan Wang, Department of Urology, Zhongnan Hospital of Wuhan University, 169 East Lake Road, Wuchang, Wuhan, Hubei 430071, P.R. China

E-mail: wangxinghuan@whu.edu.cn

Key words: WTAP, bladder cancer, relationship with prognosis

\section{Introduction}

In recent years, bladder cancer has become the most common urogenital neoplasm in China. The incidence of bladder cancer ranks first in the genitourinary cancers and it has a high recurrence rate after treatment. Therefore, it is imperative to obtain an effective method to treat this disease (1). However, ideal prognosis indicators are not yet specified (2-4) for the pathogenesis of bladder cancer and specific prognostic information is scarce, although many histological and biological indicators, such as staging and grading of cancer cells, infiltrated degree of blood vessels, size of tumor, single or multiple and deoxyribose nucleic acid (DNA) ploidy are used to evaluate the development tendency of bladder cancer.

With the development of molecular biology, biologists have studied the occurrence and developmental change rules of bladder cancer at the genetic level, which has become the study focus in recent years. The genes related to bladder cancer include the cancer gene, cancer suppressor gene and DNA mismatching repair gene $(5,6)$. Wilms tumor 1-associated protein (WTAP) is a gene closely related to occurrence and developmental change and is involved in the formation of multiple tumors, thus playing an important role. On the contrary, there are no reports on its effects in bladder cancer thus far.

Therefore, the aim of the study was to investigate the relationship between WTAP expression in bladder cancer and the recurrence and prognosis of bladder cancer, providing theoretical bases for the diagnosis, treatment and prognosis of bladder cancer.

\section{Materials and methods}

Collection of tumor tissues. Specimens were obtained from the patients who underwent surgery in the Zhongnan Hospital of Wuhan University (Wuhan, China) and had complete hospitalization data. A total of 62 fresh specimens of bladder transitional cell cancer tissues were collected as the bladder cancer group (48 males and 14 females), while 20 normal bladder mucosa specimens were selected as the control group (14 males and 6 females). The average age in the control group was $57 \pm 19$ years and that in the bladder cancer group was $52 \pm 13$ years. Through comparisons, there were no statistically 
significant differences in the sex and age between the two groups $(\mathrm{P}>0.05)$.

The study was approved by the Ethics Committee of Zhongnan Hospital of Wuhan University and informed consent was signed by the patients or guardians.

Main reagents. The main reagents used were: bicinchoninic acid (BCA) protein assay kit (Beyotime, Shanghai, China); TRIzol total ribose nucleic acid (RNA) extraction kit (Tiangen Biotech, Beijing, China); reverse transcription-polymerase chain reaction (RT-PCR) kit (Tiangen Biotech); rabbit antihuman glyceraldehyde 3-phosphate dehydrogenase (GAPDH) and WTAP polyclonal antibodies (cat. nos. 2118 and 56501, respectively; Cell Signaling Technology, Inc.; Danvers, MA, USA).

Hematoxylin and eosin $(H \& E)$ staining. Bladder tissues in the two groups were embedded into paraffin wax and made into paraffin blocks which were cut into $5-\mu \mathrm{m}$ sections as the blank sections. Then, H\&E staining was conducted using the routine histopathological method. The stained specimens were observed under light microscope (x200, Olympus Corporation, Tokyo, Japan) and then histopathological analysis was carried out.

Immunohistochemistry. Prepared tissue paraffin sections of two groups were soaked in xylene (10 min each time, 2 times), and then in the gradient ethanol for $5 \mathrm{~min}$. After the antigen repair, phosphate-buffered saline (PBS) was used to wash the resulting products (3 min each time, 3 times). Streptavidin peroxidase (SP) staining was followed by PBS washing (3 min each time, 3 times). PBS was then absorbed and normal goat serum for blocking was dripped into the sections for $15 \mathrm{~min}$ incubation at room temperature. The rabbit anti-human WTAP polyclonal antibody (1:200) were added by dripping and the wet boxes were placed into the refrigerator at $4^{\circ} \mathrm{C}$ overnight. After PBS washing (5 min each time, 3 times), SignalStain ${ }^{\circledR}$ Boost IHC Detection Reagent secondary antibody (1:600; cat. no. 8114; Cell Signaling Technology, Inc.) was added by dripping for $15 \mathrm{~min}$ incubation at $37^{\circ} \mathrm{C}$. Then, the working fluid was dripped into the foregoing product for incubation under the same conditions, after PBS washing again (5 min each time, 3 times). Then, the freshly-prepared diaminobenzidine (DAB) solution was dripped into the incubated sections and the color development degree was observed and controlled under the microscope. In addition, hematoxylin was used to re-stain the sections which were differentiated for $20 \mathrm{sec}$ by $1 \%$ hydrochloric acid alcohol to restore the blue color. Neutral gum was used to seal the section after the dehydration by gradient ethanol. All the stained specimens were observed under an Eclipse TE2000-U light microscope (x200) (Nikon Corp., Tokyo, Japan) and the analysis was performed.

RT-PCR testing. The tissues of the control group and the bladder cancer group were transferred into the Eppendorf (EP) tubes containing the RNAiso Plus extraction solution, respectively. They were settled at room temperature for $5 \mathrm{~min}$ to fully disintegrate the specimens. The specimens were then centrifuged at $12,000 \mathrm{x} \mathrm{g}$ at $4^{\circ} \mathrm{C}$. The supernatant was obtained and was added with $0.2 \mathrm{ml}$ chloroform. The supernatant was mixed evenly and settled under the same conditions as above. Afterwards, the mixed solution was centrifuged at $12,000 \mathrm{x} \mathrm{g}$ at $4^{\circ} \mathrm{C}$ for $15 \mathrm{~min}$ and the supernatant was absorbed which was added with the same volume of isopropanol. After mixing evenly, the solution was settled for $10 \mathrm{~min}$ at room temperature. Then, the solution was centrifuged under the same conditions as above for $10 \mathrm{~min}$ and the sediments were reserved with the supernatant carefully removed, and added with $1 \mathrm{ml}$ $75 \%$ ethanol. After mixing evenly, centrifugation was conducted under the same conditions for $5 \mathrm{~min}$. Similarly, the supernatant was removed and the centrifugation was repeated once. After RNA sediments were washed and the liquid was removed with the RNase-free water added. Part of total RNA solution was diluted into the $1 \mu \mathrm{g} / \mu \mathrm{l}$ solution and the reverse transcription reaction solution was prepared according to the requirements in the instructions of PrimeScript ${ }^{\circledR} \mathrm{RT}$ reagent kit with genomic DNA (gDNA) eraser and was added with the corresponding RNA specimens to conduct the reverse transcription and further obtain the complementary DNA (cDNA). The resulting cDNA was stored under $-20^{\circ} \mathrm{C}$. Subsequently, the level of mRNA was determined as indicated in the instructions of teh SYBR ${ }^{\circledR}$ Premix Ex Taq ${ }^{\mathrm{TM}}$ II (Tli RNaseH Plus) kit. The primer sequences of WTAP were: 5'-3' CAACCT CTTTAGCCAAACAAGAA and 3'-5' ATTCCTGAGTGC AACAGC.

Western blot detection. The tissues of the control and bladder cancer groups were washed with the iced normal saline and the proteins were extracted according to the protocol of the total protein extraction kit. The immunoprecipitation (IP) lysis buffer containing phenylmethanesulfonyl fluoride (PMSF) and protease inhibitor were added into the ice and the tissues were fully ground. The tissue homogenate was centrifuged at $12,000 \times \mathrm{g}$ at $4^{\circ} \mathrm{C}$ for $10 \mathrm{~min}$ and the supernatant was taken to be centrifuged under the same conditions for $20 \mathrm{~min}$. The resulting supernatant was taken. The protein content was then tested according to the protocol of the protein kit and the protein sample containing the same content of total proteins was added for sampling to conduct the sodium dodecyl sulfate polyacrylamide gel electrophoresis (SDS-PAGE) under constant voltage of $220 \mathrm{~V}$, until the bromophenol blue reached the bottom of gel. The gel was cut according to the molecular weight of the target protein and the separated proteins were electro-transferred onto the polyvinylidene fluoride (PVDF) membrane. The PVDF membrane attached with the proteins was placed in 5\% skim milk and blocked in the shaking incubator at room temperature for $3 \mathrm{~h}$. Rabbit anti-human GAPDH and WTAP polyclonal antibodies $(1: 1,000)$ were added for incubating and were placed at $4^{\circ} \mathrm{C}$ overnight. The next day, the membranes were fully washed using Tween-Tris buffered saline (TTBS) (10 min each time, 3 times) and the goat antirabbit secondary polyclonal antibody (1:2,000; cat. no. 7074; Cell Signaling Technology, Inc.) were added to incubate at room temperature for $1 \mathrm{~h}$. After TTBS washing $(10 \mathrm{~min}$ each time, 3 times), the enhanced chemiluminescence (ECL) developing solution was dripped to develop the color and then imaged.

Statistical processing. Statistical Product and Service Solutions 17.0 (SPSS 17.0) statistical software was used for 


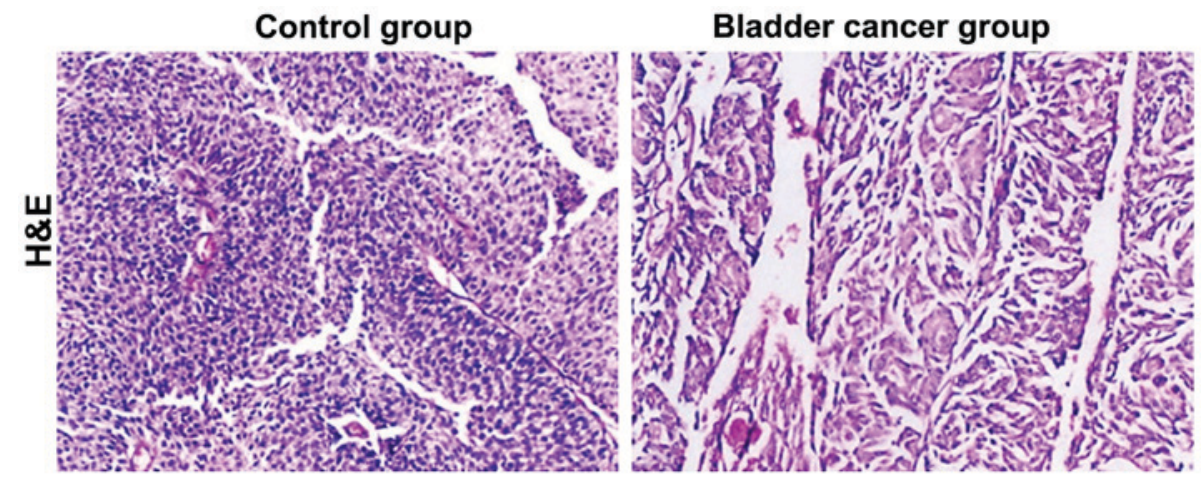

Figure 1. Hematoxylin and eosin (H\&E) staining results of tissues of the control group and the bladder cancer group (x200). Compared with tissues of the control group, those of the bladder cancer group are destroyed and the cell nuclei are damaged; moreover, a large number of inflammatory infiltrating cells with the significant histopathology is observed.

\section{Control group}

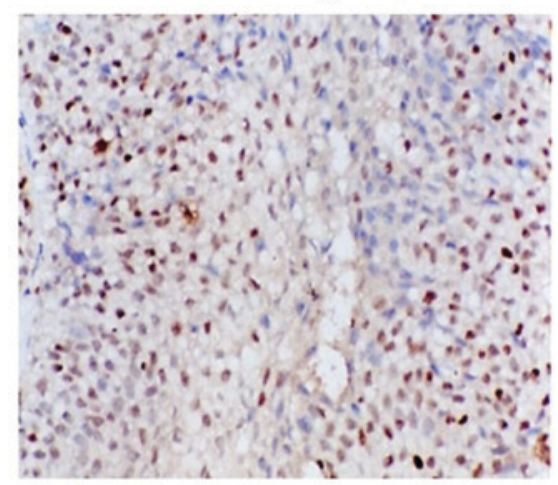

Bladder cancer group

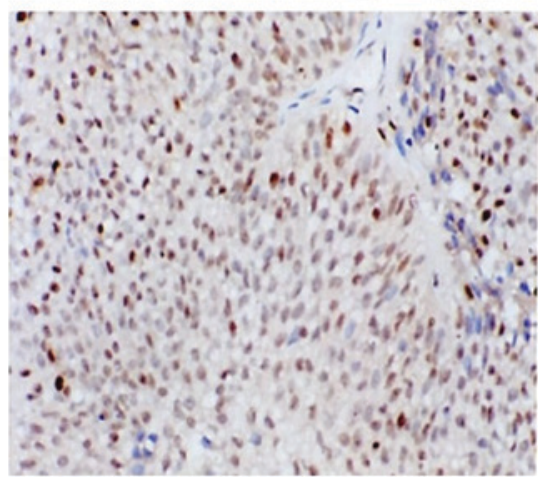

Figure 2. Immunohistochemical staining results of Wilms tumor 1-associated protein (WTAP) in tissues of the control group and the bladder cancer group (x200). WTAPs are expressed lowly in the control group, while they are expressed at a high level in the bladder cancer group.

the statistical analyses and the single factor analyses for the related data were conducted by Kaplan-Meier method. The significance of the differences was compared by the log-rank test. The effects of multiple clinical and pathological factors on the recurrence time were studied using Cox proportional hazard model. $\mathrm{P}<0.05$ was considered to indicate a statistically significant difference.

\section{Results}

Pathological conditions observed by $H \& E$ staining method. The H\&E staining sections of bladder tissues in the control and bladder cancer groups were used to specify the pathological morphology and the cell growth features of bladder cancer. Compared with tissues of the control group, those of the bladder cancer group were destroyed and the cell nuclei were damaged (Fig. 1). Moreover, there were a large number of infiltrating inflammatory cells with significant histopathology.

Immunohistochemical staining. As shown in Fig. 2, WTAP was low-expressed in the control group and brownish-yellow and tan cell nuclei were generated in the tissue sections after WTAP immunohistochemical staining. Compared with WTAP expression of the control group, that in the bladder cancer group was significantly increased.

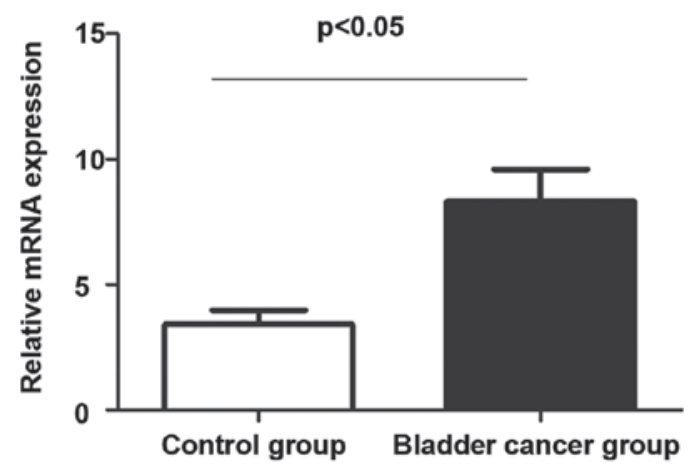

Figure 3. RT-PCR results of Wilms tumor 1-associated protein (WTAP) mRNAs in the control group and the bladder cancer group. $\mathrm{P}<0.05$ compared with the control group.

WTAP MRNA RT-PCR results in the control group and the bladder cancer group. The tissue specimens of the control and bladder cancer groups were extracted, respectively. Then, RT-PCR was conducted for the total RNA extracted for the two groups. According to results, the expression of WTAP in the control group was significantly lower than that in the bladder cancer group and this indicated that there were a large number of WTAP mRNAs transcribed in the bladder cancer group (Fig. 3). 

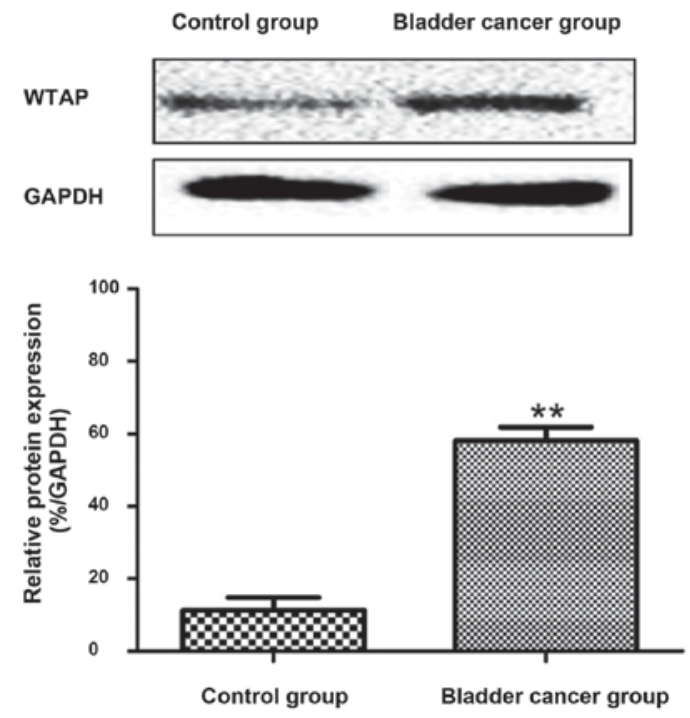

Figure 4. Western blot results of Wilms tumor 1-associated proteins (WTAPs) in the control group and the bladder cancer group. ${ }^{* *} \mathrm{P}<0.01$ compared with the control group.

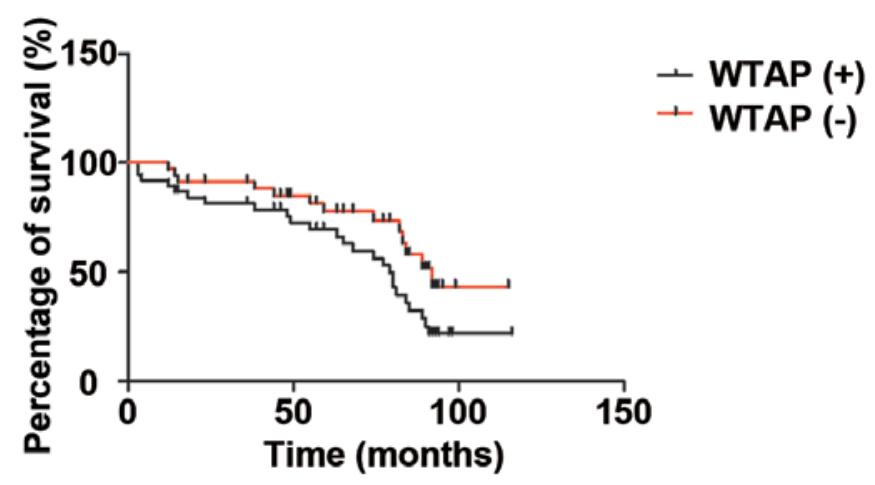

Figure 5. The relationship between Wilms tumor 1-associated protein (WTAP) and the prognosis of bladder cancer patients. The total survival period in the negative WTAP expression group is significantly higher than that in the positive WTAP expression group.

Western blot analysis of WTAP proteins in the control and bladder cancer groups. According to western blot results, WTAP proteins were poorly expressed in the bladder tissues of the control group, while the expression was high in the tissues of the bladder cancer group (Fig. 4).

Relationship between WTAP and the prognosis of bladder cancer patients. The Kaplan-Meier survival analyses were conducted to show the effects of both the negative and positive WTAP expression levels on the prognosis of patients. Fig. 5 shows that the total survival period in the negative WTAP expression group was significantly higher than that in the positive WTAP expression group $(\mathrm{P}<0.05)$.

\section{Discussion}

The occurrence and development of bladder cancer are the results of multiple cancer genes and cancer suppressor genes simultaneously or sequentially acting in the same environment. In recent years, the incidence of bladder cancer is rising year by year world-wide affecting both adult and young people $(7,8)$. Bladder cancer refers to a common malignant tumor that generally occurs in the bladder mucosa, and is the most common tumor in the genitourinary system (9-11). People at different ages, both female and male, can be affected by bladder cancer, but males and the elderly are more vulnerable to this disease which severely harms human health (12-14). Therefore, it is crucial to identify an effective method to treat bladder cancer. A number of studies have previously been carried out to determine the diagnosis and treatment of bladder cancer (15-17). WTAP is a key gene involving many tumor diseases. Previous findings have shgown that WTAP is involved and plays an important role in the occurrence and development of tumors (18-20). However, the effects of WTAP in bladder cancer should be studied further and its molecular mechanisms should be also verified.

WTAP, which is a key regulatory factor, is thought to be closely related to the occurrence and development of many kinds of malignant tumors (21). WTAP can promote the occurrence and development of malignant tumor by stabilizing $\mathrm{C}-\mathrm{Myc}$, promoting cell proliferation and anchoring independent growth, blocking senescence and differentiation (22). In this study, we conducted a preliminary study on whether WTAP can be used as a diagnostic marker for bladder cancer. Our results showed that the cell structure was destroyed and the nucleus shrank in the bladder cancer group, while the tissue in the control group was intact. Immunohistochemical staining showed that the expression of WTAP in bladder cancer group was significantly higher than that in control group. This suggests that the expression of WTAP in bladder tumors is more specific. Therefore, WTAP may be used as a new tumor marker in the diagnosis of bladder tumors. At the same time, as a therapeutic target, further research on WTAP is needed. The results of RT-PCR and Western blot analysis showed that WTAP mRNA and protein were highly expressed in bladder cancer group.

In conclusion, the present study proved that WTAP expression in the bladder cancer group was significantly increased compared with that in the control group, indicating that WTAP may play an important role in the occurrence and development of bladder cancer. This provides a new thought and direction for the diagnosis and treatment of bladder cancer and is expected to be a new approach for the treatment of bladder cancer patients in the future.

\section{Acknowledgements}

Not applicable.

\section{Funding}

No funding was received.

\section{Availability of data and materials}

The datasets used and/or analyzed during the current study are available from the corresponding author on reasonable request. 


\section{Authors' contributions}

LC analyzed HE staining result. XW performed immunohistochemistry. LC wrote and XW revised the manuscript. Both authors read and approved the final manuscript.

\section{Ethics approval and consent to participate}

The study was approved by the Ethics Committee of Zhongnan Hospital of Wuhan University (Wuhan, China) and informed consent was signed by the patients or guardians.

\section{Patient consent for publication}

Not applicable.

\section{Competing interests}

The authors declare that they have no competing interests.

\section{Authors' information (optional)}

Not applicable.

\section{References}

1. Gupta S, Hau AM, Beach JR, Harwalker J, Mantuano E, Gonias SL, Egelhoff TT and Hansel DE: Mammalian target of rapamycin complex 2 (mTORC2) is a critical determinant of bladder cancer invasion. PLoS One 8: e81081, 2013.

2. Cairns P, Evron E, Okami K, Halachmi N, Esteller M, Herman JG, Bose S, Wang SI, Parsons R and Sidransky D: Point mutation and homozygous deletion of PTEN/MMAC1 in primary bladder cancers. Oncogene 16: 3215-3218, 1998.

3. Bellmunt J, Albiol S and Kataja V; ESMO Guidelines Working Group: Invasive bladder cancer: ESMO clinical recommendations for diagnosis, treatment and follow-up. Ann Oncol 20 (Suppl 4): 79-80, 2009.

4. Fiebig HH, Dengler AW and Roth T: Human tumor xenografts: predictivity, characterization and discovery of new anticancer agents. In: Contributions to Oncology. Relevance of Tumor Models for Anticancer Drug Development. Fiebig $\mathrm{HH}$ and Burger AM (eds). Vol. 54. Contr Oncol Basel, Karger, pp29-50, 1999.

5. El-Kott AF, Khalil AM and El-Kenawy A-M: Immunohistochemical expressions of uPA and its receptor UPAR and their prognostic significant in urinary bladder carcinoma. Int Urol Nephrol 36: 417-423, 2004.

6. Span PN, Witjes JA, Grebenchtchikov N, Geurts-Moespot A, Moonen PM, Aalders TW, Vriesema JL, Kiemeney LA, Schalken JA and Sweep FC: Components of the plasminogen activator system and their complexes in renal cell and bladder cancer: Comparison between normal and matched cancerous tissues. BJU Int 102: 177-182, 2008.

7. Richter J, Beffa L, Wagner U, Schraml P, Gasser TC, Moch H, Mihatsch MJ and Sauter G: Patterns of chromosomal imbalances in advanced urinary bladder cancer detected by comparative genomic hybridization. Am J Pathol 153: 1615-1621, 1998.
8. Bruch J, Wöhr G, Hautmann R, Mattfeldt T, Brüderlein S, Möller P, Sauter S, Hameister H, Vogel W and Paiss T: Chromosomal changes during progression of transitional cell carcinoma of the bladder and delineation of the amplified interval on chromosome arm 8q. Genes Chromosomes Cancer 23: 167-174, 1998.

9. Lindblad-Toh K, Tanenbaum DM, Daly MJ, Winchester E, Lui WO, Villapakkam A, Stanton SE, Larsson C, Hudson TJ Johnson BE, et al: Loss-of-heterozygosity analysis of small-cell lung carcinomas using single-nucleotide polymorphism arrays Nat Biotechnol 18: 1001-1005, 2000.

10. Jänne PA, Li C, Zhao X, Girard L, Chen TH, Minna J, Christiani DC, Johnson BE and Meyerson M: High-resolution single-nucleotide polymorphism array and clustering analysis of loss of heterozygosity in human lung cancer cell lines. Oncogene 23: 2716-2726, 2004.

11. Suraweera N, Duval A, Reperant M, Vaury C, Furlan D, Leroy K, Seruca R, Iacopetta B and Hamelin R: Evaluation of tumor microsatellite instability using five quasimonomorphic mononucleotide repeats and pentaplex PCR. Gastroenterology 123: 1804-1811, 2002

12. Bonnal C, Ravery V, Toublanc M, Bertrand G, Boccon-Gibod L, Hénin D and Grandchamp B: Absence of microsatellite instability in transitional cell carcinoma of the bladder. Urology 55: 287-291, 2000

13. Iyer G, Al-Ahmadie H, Schultz N, Hanrahan AJ, Ostrovnaya I, Balar AV, Kim PH, Lin O, Weinhold N, Sander C, et al: Prevalence and co-occurrence of actionable genomic alterations in high-grade bladder cancer. J Clin Oncol 31: 3133-3140, 2013.

14. Hudson MA and McReynolds LM: Urokinase and the urokinase receptor: Association with in vitro invasiveness of human bladder cancer cell lines. J Natl Cancer Inst 89: 709-717, 1997.

15. Russell PJ, Raghavan D, Gregory P, Philips J, Wills EJ, Jelbart M, Wass J, Zbroja RA and Vincent PC: Bladder cancer xenografts: A model of tumor cell heterogeneity. Cancer Res 46: 2035-2040, 1986.

16. Russell PJ, Raghavan D, Philips J and Gregory P: Applications of the xenograft as a model of invasive transitional cell carcinoma of the bladder. Prog Clin Biol Res 260: 167-181, 1988.

17. Kovnat A, Armitage M and Tannock I: Xenografts of human bladder cancer in immune-deprived mice. Cancer Res 42: 3696-3703, 1982.

18. Kovnat A, Buick RN, Connolly JG, Jewett MA, Keresteci AG and Tannock IF: Comparison of growth of human bladder cancer in tissue culture or as xenografts with clinical and pathological characteristics. Cancer Res 44: 2530-2533, 1984.

19. Zhang H, Aina OH, Lam KS, de Vere White R, Evans C, Henderson P, Lara PN, Wang X, Bassuk JA and Pan CX: Identification of a bladder cancer-specific ligand using a combinatorial chemistry approach. Urol Oncol 30: 635-645, 2012.

20. Lin TY, Li YP, Zhang H, Luo J, Goodwin N, Gao T, White RV, Lam KS and Pan CX: Tumor-targeting multifunctional micelles for imaging and chemotherapy of advanced bladder cancer. Nanomedicine (Lond) 8: 1239-1251, 2013.

21. Anderson AM, Weasner BP, Weasner BM and Kumar JP: The Drosophila Wilms' Tumor 1-Associating Protein (WTAP) homolog is required for eye development. Dev Biol 390: 170-180, 2014.

22. Horiuchi K, Kawamura T, Iwanari H, Ohashi R, Naito M, Kodama $\mathrm{T}$ and Hamakubo T: Identification of Wilms' tumor 1-associating protein complex and its role in alternative splicing and the cell cycle. J Biol Chem 288: 33292-33302, 2013.

This work is licensed under a Creative Commons Attribution-NonCommercial-NoDerivatives 4.0 International (CC BY-NC-ND 4.0) License. 